\title{
Circulating Metabolomic Profiling Detects Novel Markers of Myocardial Infarction among Cardiac-chest Pain Cases
}

\author{
$\mathrm{Nan} \mathrm{Aa}$ \\ Department of Cardiology \\ Ying Lu \\ Department of Laboratory \\ Mengjie Yu \\ Jiangsu Province Key Laboratory of Drug Metabolism and Pharmacokinetics \\ Heng Tang \\ Department of Cardiology, the First Affiliated Hospital of Nanjing Medical University \\ Zhenyao Lu \\ Laboratory of Metabolomics, Jiangsu Province Key Laboratory of Drug Metabolism and Pharmacokinetics \\ Runbin Sun \\ Laboratory of Metabolomics, Jiangsu Province Key Laboratory of Drug Metabolism and Pharmacokinetics

\section{Liansheng Wang} \\ the First Affiliated Hospital of Nanjing Medical University \\ Chunjian Li \\ Department of Cardiology \\ Zhijian Yang \\ Department of Cardiology \\ Jiye Aa ( $\nabla$ jiyea@cpu.edu.cn ) \\ China Pharmaceutical University

\section{Xiangqing Kong} \\ Department of Cardiology

\section{Guangji Wang} \\ Laboratory of Metabolomics, Jiangsu Province Key Laboratory of Drug Metabolism and Pharmacokinetics
}

\section{Research}

Keywords: Plasma, Myocardial infarction, Metabolites, Biomarker, Transcriptomics

Posted Date: December 2nd, 2020

DOI: https://doi.org/10.21203/rs.3.rs-117484/v1

License: (c) (i) This work is licensed under a Creative Commons Attribution 4.0 International License. Read Full License 


\section{Abstract}

Background: A unique human plasma metabolic pattern of myocardial infarction (MI) has been recognized for over a decade. The discriminant metabolites are potential diagnosis and warning markers, and have connections with traditional cardiovascular risk factors and circulating transcriptomics.

Methods: A total of 146 chest pain ( 85 Mls and 61 non-Ml chest pain cases) and 84 control individuals were recruited. GC/MS and LC/MS were applied to identify and quantify the plasma metabolites. The metabolites were analyzed by multivariate statistical analysis to describe different patterns of CADs with chest pain. Receiver operating characteristic $\triangle \mathrm{ROC} \bigotimes$ and odds ratio (OR) were calculated to identify potential markers of MI cases, and the markers were further evaluated based on human circulating transcriptomics from the GEO database. The correlation of metabolites to clinical indices was assessed.

Results: MI can be clearly distinguished from non-MI chest pain cases by the metabolites we identified, better than by medical history combined with routine biochemical parameters. Pathway analysis highlighted an upregulated methionine metabolism and downregulated arginine biosynthesis that were deeply involved in MI cases. Among all the metabolites, deoxyuridine (dU) and methionine scored highly in ROC (>0.91) and are related to LDH and AST $(p<0.05)$. OR values (logOR>2) suggested, with or without adjusting by lipid levels, age, smoking, diabetes and hypertension, that dU and methionine are risk factors for alerting about MI occurrence. Transcriptomics data showed elevations of plasma CDA and UPP1, and a decrease of cardiac MARS, which are consistent with the increased levels of plasma dU and methionine, respectively.

Conclusions: For the first time, dU and methionine were suggested as markers for MI diagnosis and as high-risk markers to alert about an onset of MI. Plasma metabolite changes are not only directly related to cardiac damage but also could be related to circulating cells.

\section{Background}

A strangling feeling in the chest is a typical manifestation of coronary artery disease (CAD). In most cases, CAD develops as plaque builds up on the artery walls. When it progresses to myocardial infarction (MI), coronary heart disease will be life-threatening and extremely dangerous in the ensuing days or weeks due to its various fatal complications. However, chest pain can be caused by other cardiovascular events (e.g., unstable angina) or other, non-cardiac cases (e.g., gastroesophageal reflux disease). Thus, noticing early warning signs to alert patients about the onset of Ml, taking precautions and recognizing $\mathrm{Ml}$ are among the primary focuses of the management of atherosclerotic coronary artery disease, which is the main cause of coronary heart diseases.

Diagnostic and predictive biomarkers of MI are of crucial importance. Among all cardiac damage biomarkers, such as CK-MB, AST and cardiac troponins (cTnt), cTnt is the preferred MI biomarker clinically. However, some studies showed that an unexpected elevation of this cardiomyocyte protein was observed without obvious connection to cardiac injury. Hence, clinicians need to carefully determine whether troponin elevation is direct proof of myocardial necrosis, especially when the underlying reason for its release is unclear. [1-3]

More potential biomarkers are urgently needed to facilitate the diagnosis of, as well as for preferably alerting about and predicting MI onset. CAD is a lifestyle disease, and the abnormal abundance of circulating metabolites surely reflects the metabolic state of CAD patients. Medical history records and laboratory tests, e.g., sex, age, smoking history, function of the primary organs, and biochemical assays, are important factors worthy of consideration in identifying diagnostic markers and clarifying the underlying mechanism involved. Since ischemic heart diseases are characterized by profound metabolic shifts at both the circulatory and local levels[4], metabolomics has been applied to study the metabolic pattern changes detected in the plasma or serum of CAD patients. Early in 2002, a pioneering work was published showing that NMR-based metabonomics had the potential to rapidly and noninvasively diagnose the presence and severity of coronary heart disease[5]. In 2005, Marc Sabatine and his colleagues identified metabolic biomarkers of myocardial ischemia associated with physical exercise[6]. Later studies focused on identifying biomarkers and metabolic pathways and exploring the underlying mechanisms associated with cardiovascular diseases[7-13]. Excitingly, a panel of potential markers has been suggested for coronary heart diseases, such as arginine and homocysteine, and the underlying mechanisms of their action have been explored[14-16]. Unfortunately, few publications have focused on the diagnosis and prediction of $\mathrm{MI}$ onset among CAD patients with chest pains, although the occurrence of $\mathrm{MI}$ is deeply influenced by whether an individual has traditional risk factors, such as male sex, diabetes, tobacco smoking and hypertension.

In this study, a metabolomic platform with GC/MS and LC/MS instrumentation was employed to profile plasma metabolites of hospitalized CAD patients with chest pain (including $\mathrm{MI}$ and non-MI chest pain cases) and their controls. Metabolic patterns were evaluated, and metabolic markers were screened and described based on semi-quantitative data, AUC, odds ratios (OR)[17] and their connections with well-recognized cardiovascular disease risk factors We aimed to identify metabolic markers to facilitate the diagnosis and early prediction of $\mathrm{Ml}$ among chest pain patients. The mechanisms behind the biomarker changes are also briefly discussed.

\section{Methods}

\subsection{Human plasma samples}

A total of 146 chest pain cases highly suspected of Ml, and 84 individual controls were recruited from October, 2017 to March, 2018 , with exclusions criteria of fever, hepatic decompensation, renal failure, cancers, endocrine and hematological diseases. The blood samples of chest pain cases were 
collected within 48 hours since the symptom was first reported and fasting for at least 6 hours. All control cases were fasting for at least 6 hours too. After routine diagnostic procedures, including evidence from ECGs, serum infarction biomarkers, computed tomography angiography or coronary angiography, 85 were later confirmed to be NSTEMI or STEM as the MI cases (MIs) and the remaining 61 were confirmed to be non-MI chest pain cases (non-MIs). All the non-Mls include 34 unstable angina (UA) cases, and 27 other non-MI cardiac cases (non-MICs), including myocarditis, valvular heart diseases etc.

The venous blood samples were collected from fasting state volunteers in EDTA-Na anticoagulated tubes in the morning. Within 2 hours, blood samples were centrifuged at $1000 \mathrm{~g}$ for $5 \mathrm{~min}$, and each of the supernatant plasma was transferred to another tube, frozen at $-80^{\circ} \mathrm{C}$ in refrigerator. Before use of the plasma samples, they were thawed by incubation at $37^{\circ} \mathrm{C}$ bath for $15 \mathrm{~min}$, vortexed and centrifuged at $650 \mathrm{~g}$ for $5 \mathrm{~min}$.

\subsection{Equipment for blood examinations}

The instrument blood count and blood biochemistry, model xn-10, is made in Hyogo, Japan. Both NT-proBNP and serum infarction marker (cTns) are Roche company of Germany, model Cobas 6000, produced in Mannheim, Germany. The biochemical instrument is Beckman Coulter of the United States, model au5800, produced in Shizuoka, Japan.

\subsection{Chemicals and reagents}

Stable isotope internal standard $5-{ }^{13} \mathrm{C}$-glutamine was purchased from Cambridge Isotope Laboratories (Andover, MA, USA). Myristic-1,2- ${ }^{13} \mathrm{C} 2$ acid, methoxamine hydrochloride (purity $98 \%$ ) and pyridine ( $\geq 99.8 \% \mathrm{GC}$ ) were purchased from Sigma-Aldrich (St. Louis, MO, USA). N-methyl-trimethylsilyltrifluoroacetamide (MSTFA) plus 1\% trimethylchlorosilane (TMCS) were provided by Pierce Chemical (Rockford, IL, USA). Methanol, acetonitrile and nheptane were HPLC grade and obtained from Merck (Darmstadt, Germany). Purified water was produced by a Milli-Q system (Millipore, Bedford, MA, USA). Ammonium acetate (purity $98.0 \%$ ) and ammonia solution (25\%, w/w) were purchased from Aladdin (Shanghai, China) and Nanjing Chemical Reagent (Nanjing, China), respectively.

\subsection{GC/MS and UPLC-QTOF/MS analysis}

A well-developed metabolomic platform was utilized to profile metabolites in plasma samples of the clinic patients. For the analysis in GC/MS system((Shimadzu GCMS-QP2010 Ultra, Kyoto, Japan), the plasma samples were pretreated, extracted, and derivatised in a similar way to that described previously[18], Method S1. To minimize systematic variations, the samples were randomly analyzed with the quality control (QC) samples inserted, and the intensity of internal standard(IS), myristic-1,2-13 $\mathrm{C}_{2}$ acid, and an external reference standard, methyl myristate was evaluated. The raw GC/MS data were processed using a post-run analysis software(Shimadzu GC/MS solution 2.0), each of the metabolite was identified and the quantitative data was acquired in the same way as reported[19].

For the analysis in UPLC-QTOF/MS system (AB SCIEX TripleTOF® 5600, Foster City, CA), the plasma samples were pretreated in the same way as in $\mathrm{GC} / \mathrm{MS}$ analysis with a few modifications, which used the other internal standard of $5{ }^{-13} \mathrm{C}$-glutamine dissolved in methanol at $15 \mu \mathrm{g} / \mathrm{ml}$. The UPLCQTOF/MS analysis was carried out as previously reported[20]. The detail information and protocol for GC/MS and UPLC-QTOF/MS is available in the Supplementary material, method S2.

\subsection{Multivariate statistical analysis}

After acquirement of the data in GC/MS and UPLC-QTOF/MS,the detected peaks/identified metabolites in each sample were aligned, quantitated, and all the samples data constructed a data matrix, where the missing data were excluded as default during analysis. After normalization against the IS, the data was evaluated using SIMCA P14.1 software (Umetrics, Umeå, Sweden) [19], Method S3. Concisely, principal component analysis(PCA), partial least square to latent structure discriminant analysis (PLS-DA), orthogonal PLS-DA(OPLS-DA) models were calculated to show the clustering or separation of samples from different groups For PLS-DA modeling, samples from the different groups were classified such that all samples were divided into different groups (e.g., Mls, non-Mls, controls, etc.) as the qualitative 'dummy' variables, Y. The goodness of fit for the models was evaluated using three quantitative parameters: $R^{2} X$ and $R^{2} Y$ were the explained variation in $X$ and $Y$, respectively, and $Q^{2} Y$ was the predicted variation in $Y$. Permutation test was assessed for model validation, where the higher level of $R^{2} Y$ and $Q^{2} Y$, and lower value of the intercept of $R^{2}$ (lower than 0.2 ) and $Q^{2}$ (lower than 0.0 ) suggested good model and prediction ability.

\subsection{Discriminant metabolites and statistical analysis}

After normalization against the IS, all the semi-quantitative data from both GC/MS and UPLC-QTOF/MS was logarized so that the state probabilities of the data queue tended to a normal distribution. The discriminant metabolites between groups were screened and chosen based on Variable Importance (VIP) using SIMCA-P 14.1, and the independent sample t-test of the logarized data using SPSS (version 23.0, SPSS Inc., Chicago, IL, US).

Metabolic pathway enrichment and topology analyses were performed using Metaboanalyst 3(https://www.metaboanalyst.ca/), Method S4. The KEGG ID of discriminatory compounds were uploaded and embedded in human pathway library for pathway analysis and hypergeometric tests, with the pathway analysis algorithms of Fisher's Exact Test, a pathway topology algorithms of Relative-betweeness Centrality, and KEGG pathway library version of Homo sapiens. Potential biomarkers were identified based on the identified metabolic pathways in databases such as KEGG (http://www.genome.jp/kegg/), human metabolomic data base (HMDB), (http://www.hmdb.ca/) and LipidMaps (http://www.lipidmaps.org/). 
For the data inconformity with normal distribution from clinical assaying, a nonparametric test (Mann-Whitney U test, two-sided) was employed to evaluate statistical significance. ROC analysis and OR calculation were performed using SPSS as well. Before computing OR value, the logarized data of a metabolite(i) for each subject of ORi was normalized by subtracting mean value of ORmean within this group, and then divided by the standard deviation(SD) within the group, shown as the normalized ORs=(ORi-ORmean)/SD.

\subsection{Transcriptomics data}

We studied open access transcriptomics data from GEO. The human myocardial infarction plasma data are from GEO accession GSE48060. Mice myocardial infarction data are from GSM12346. Date were directly plotted without transformation.

\section{Results}

\subsection{Clinical description of chest pain individuals by PCA and OPLS-DA plots}

Tables 1 and 2 show the history examinations and basic laboratory tests of the volunteers. Generally, higher glucose, AST, LDH, HBDH, and CK levels and lower ALB and $\mathrm{Ca}^{2+}$ concentrations were detected in the chest pain patients than the control group. Among the 146 chest pain inpatients, approximately $65 \%$ had taken aspirin and statin treatments before blood collection. As a result, TC, TG, LDL-c and HDL-c levels were all lower in chest pain inpatients than the controls. 
Table 1

Sample Characteristics: Controls vs all the chest pain cases

\begin{tabular}{|c|c|c|c|c|}
\hline Clinical concerns & Variables & The controls & The chest pain cases & Statistics \\
\hline & & $\mathrm{n}=84$ & $n=146$ & p Values \\
\hline \multirow[t]{2}{*}{ Demographics } & Male & 56 & 99 & 0.26 \\
\hline & Age(years) & $50.25 \pm 1.76$ & $59.28 \pm 1.83$ & 0.00 \\
\hline \multirow[t]{9}{*}{ Cardiac risk factors } & Hypertension & 32 & 83 & 0.00 \\
\hline & Diabetes & 8 & 36 & 0.00 \\
\hline & $\mathrm{TC}(\mathrm{mmol} / \mathrm{L})$ & $4.93 \pm 0.15$ & $4.08 \pm 0.11$ & 0.00 \\
\hline & $\mathrm{TG}(\mathrm{mmol} / \mathrm{L})$ & $1.43 \pm 0.12$ & $1.51 \pm 0.08$ & 0.17 \\
\hline & LDL-C(mmol/L) & $3.15 \pm 0.11$ & $2.70 \pm 0.08$ & 0.00 \\
\hline & $\mathrm{HDL}-\mathrm{C}(\mathrm{mmol} / \mathrm{L})$ & $1.31 \pm 0.04$ & $0.95 \pm 0.02$ & 0.00 \\
\hline & $\mathrm{LPa}(\mathrm{mg} / \mathrm{L})$ & $349.35 \pm 51.45$ & $339.39 \pm 27.47$ & 0.65 \\
\hline & Tobacco using & 8 & 43 & 0.00 \\
\hline & Drinking history & 0 & 16 & 0.00 \\
\hline \multirow[t]{3}{*}{ Cardiovascular medications } & Aspirin & 1 & 88 & 0.00 \\
\hline & Statin therapy & 6 & 94 & 0.00 \\
\hline & $\beta$-blockers & 0 & 7 & 0.00 \\
\hline Prior cardiovascular disease & & 2 & 22 & 0.01 \\
\hline \multirow[t]{19}{*}{ Biochemical items } & $\operatorname{ALT}(\mathrm{U} / \mathrm{L})$ & $27.15 \pm 2.48$ & $39.33 \pm 2.90$ & 0.00 \\
\hline & AST(U/L) & $26.31 \pm 1.45$ & $77.35 \pm 9.78$ & 0.00 \\
\hline & $\operatorname{ALP}(U / L)$ & $78.20 \pm 2.19$ & $86.16 \pm 2.26$ & 0.03 \\
\hline & $\mathrm{GGT}(\mathrm{U} / \mathrm{L})$ & $30.69 \pm 2.75$ & $45.18 \pm 3.80$ & 0.02 \\
\hline & $\mathrm{LDH}(\mathrm{U} / \mathrm{L})$ & $169.30 \pm 3.85$ & $398.72 \pm 32.22$ & 0.00 \\
\hline & $\mathrm{CK}(\mathrm{U} / \mathrm{L})$ & $119.30 \pm 11.27$ & $480.97 \pm 78.58$ & 0.94 \\
\hline & $\mathrm{HBDH}(\mathrm{U} / \mathrm{L})$ & $107.80 \pm 3.87$ & $297.38 \pm 28.85$ & 0.00 \\
\hline & TBIL $(\mu \mathrm{mol} / \mathrm{L})$ & $14.36 \pm 0.56$ & $13.74 \pm 0.71$ & 0.03 \\
\hline & $\mathrm{DBIL}(\mu \mathrm{mol} / \mathrm{L})$ & $4.51 \pm 0.28$ & $4.96 \pm 0.26$ & 0.83 \\
\hline & $\mathrm{IBIL}(\mu \mathrm{mol} / \mathrm{L})$ & $9.16 \pm 0.42$ & $8.77 \pm 0.48$ & 0.05 \\
\hline & $\mathrm{TP}(\mathrm{g} / \mathrm{L})$ & $70.80 \pm 0.47$ & $61.12 \pm 0.48$ & 0.00 \\
\hline & $\operatorname{ALB}(\mathrm{g} / \mathrm{L})$ & $44.68 \pm 0.54$ & $35.80 \pm 0.36$ & 0.00 \\
\hline & $\mathrm{GLB}(\mathrm{g} / \mathrm{L})$ & $25.78 \pm 0.48$ & $25.33 \pm 0.39$ & 0.14 \\
\hline & ALB/GLB & $1.79 \pm 0.05$ & $1.45 \pm 0.03$ & 0.00 \\
\hline & $\mathrm{GLU}(\mathrm{mmol} / \mathrm{L})$ & $5.77 \pm 0.13$ & $6.01 \pm 0.22$ & 0.23 \\
\hline & Urea(mmol/L) & $5.13 \pm 0.18$ & $7.37 \pm 0.52$ & 0.00 \\
\hline & $\operatorname{Cr}(\mu \mathrm{mol} / \mathrm{L})$ & $71.14 \pm 2.00$ & $102.13 \pm 11.16$ & 0.00 \\
\hline & $\mathrm{UA}(\mu \mathrm{mol} / \mathrm{L})$ & $325.57 \pm 9.61$ & $378.91 \pm 13.24$ & 0.04 \\
\hline & $\mathrm{Ca}(\mathrm{mmol} / \mathrm{L})$ & $2.36 \pm 0.02$ & $2.17 \pm 0.01$ & 0.00 \\
\hline
\end{tabular}


Table 2

Sample Characteristics: MI vs non-MI chest pain cases

\begin{tabular}{|c|c|c|c|c|c|}
\hline \multirow[t]{2}{*}{ Clinical concerns } & \multirow[t]{2}{*}{ Variables } & \multirow{2}{*}{$\begin{array}{l}\text { Controls } \\
(n=84)\end{array}$} & \multicolumn{2}{|l|}{ Chest pain cases } & \multirow{2}{*}{$\begin{array}{l}\text { Statistics } \\
p \text { values }\end{array}$} \\
\hline & & & non-MI(n = 61) & $M I(n=85)$ & \\
\hline \multirow[t]{2}{*}{ Demographics } & Male & 56 & 32 & 67 & 0.00 \\
\hline & Age(years) & $50.25 \pm 1.76$ & $60.18 \pm 2.42$ & $65.08 \pm 1.60$ & 0.12 \\
\hline \multirow[t]{8}{*}{ Cardiac risk factors } & Hypertension & 32 & 32 & 51 & 0.28 \\
\hline & Diabetes & 8 & 8 & 28 & 0.01 \\
\hline & Tobacco using & 8 & 8 & 35 & 0.00 \\
\hline & $\mathrm{TC}(\mathrm{mmol} / \mathrm{L})$ & $4.93 \pm 0.15$ & $3.69 \pm 0.12$ & $4.28 \pm 0.15$ & 0.01 \\
\hline & $\mathrm{TG}(\mathrm{mmol} / \mathrm{L})$ & $1.43 \pm 0.12$ & $1.26 \pm 0.09$ & $1.65 \pm 0.11$ & 0.02 \\
\hline & LDL-C(mmol/L) & $3.15 \pm 0.11$ & $2.40 \pm 0.09$ & $2.86 \pm 0.11$ & 0.01 \\
\hline & $\mathrm{HDL}-\mathrm{C}(\mathrm{mmol} / \mathrm{L})$ & $1.31 \pm 0.04$ & $0.96 \pm 0.03$ & $0.94 \pm 0.03$ & 0.70 \\
\hline & $\mathrm{LPa}(\mathrm{mg} / \mathrm{L})$ & $349.35 \pm 51.45$ & $334.55 \pm 47.64$ & $341.96 \pm 33.82$ & 0.56 \\
\hline \multicolumn{2}{|c|}{ Prior cardiovascular disease } & 2 & 12 & 10 & 0.24 \\
\hline \multirow[t]{3}{*}{ Serum biomarkers } & cTNT(ng/ml) & - & $756.13 \pm 242.59$ & $1559.30 \pm 276.64$ & 0.56 \\
\hline & CK-MB(U/L) & - & $24.98 \pm 6.08$ & $32.10 \pm 5.89$ & 0.68 \\
\hline & $\mathrm{Mb}(\mathrm{ng} / \mathrm{ml})$ & - & $59.30 \pm 23.79$ & $66.99 \pm 15.32$ & 0.46 \\
\hline \multirow[t]{8}{*}{ Biochemical items } & $\operatorname{ALT}(\mathrm{U} / \mathrm{L})$ & $27.15 \pm 2.48$ & $32.17 \pm 4.59$ & $43.14 \pm 3.65$ & 0.04 \\
\hline & AST(U/L) & $26.31 \pm 1.45$ & $29.12 \pm 2.91$ & $102.99 \pm 14.10$ & 0.00 \\
\hline & $\mathrm{LDH}(\mathrm{U} / \mathrm{L})$ & $169.30 \pm 3.85$ & $213.05 \pm 10.22$ & $497.43 \pm 45.36$ & 0.00 \\
\hline & $\mathrm{CK}(\mathrm{U} / \mathrm{L})$ & $119.30 \pm 11.27$ & $86.83 \pm 12.99$ & $690.51 \pm 113.49$ & 0.00 \\
\hline & $\mathrm{HBDH}(\mathrm{U} / \mathrm{L})$ & $107.80 \pm 3.87$ & $132.79 \pm 6.71$ & $384.89 \pm 40.81$ & 0.00 \\
\hline & $\mathrm{TP}(\mathrm{g} / \mathrm{L})$ & $70.80 \pm 0.47$ & $61.95 \pm 0.71$ & $60.68 \pm 0.62$ & 0.18 \\
\hline & $\mathrm{GLU}(\mathrm{mmol} / \mathrm{L})$ & $5.77 \pm 0.13$ & $5.49 \pm 0.22$ & $6.28 \pm 0.32$ & 0.06 \\
\hline & $\mathrm{Ca}(\mathrm{mmol} / \mathrm{L})$ & $2.36 \pm 0.02$ & $2.20 \pm 0.02$ & $2.16 \pm 0.02$ & 0.11 \\
\hline
\end{tabular}

Based on clinical parameters (listed in Table 1 "variables"), including "biochemical items", "demographics" and "cardiac risk factors", an unsupervised PCA score plot was created. The model indicated a few outliers when the samples were either divided into 3 (controls, Mls, non-Mls) or 4 groups (controls, UA, MIs and other non-MICs), and each of the groups generally overlapped with the others (Fig. 1A1, 1A2). However, a supervised PLS-DA revealed a visible separation of the groups with only a little overlap when the samples were divided into 3 groups, i.e., Mls, non-Mls and controls. When the samples were divided into 4 groups (MIs, UAs, other non-MICs, and controls), the controls, UAs and Mls were fairly well separated, but the other nonMICs primarily showed overlaps with UAs and MIs (Fig. 1A3, 1A4). These findings suggest that the model was not powerful at differentiating non-MICs from Mls and UAs based on basic laboratory tests and history examinations.

\subsection{Plasma metabolomic description of chest pain individuals by PCA and OPLS-DA plots}

GC/MS and LC/MS analysis of the plasma samples aligned the metabolites in typical chromatograms (Figure S1-2). Deconvolution of the GC/MS chromatograms produced 135 independent peaks from the plasma samples, 83 of which were authentically identified as metabolites; LC/MS produced 279 peaks, and 76 metabolites were identified (Table S1-2). Quantitative data were acquired for each metabolite in the plasma samples of the control, MI, UA and other non-MI cardiac cases.

Based on the metabolomic data derived from GC/MS and LC/MS analysis, the PCA score plot again showed a few outliers when the samples were divided into 3 or 4 groups, as indicated above. Unlike with the clinical data, unsupervised PCA analysis of metabolomic data showed that the majority of Mls deviated from the others, regardless of whether the 3 or 4 groups were defined, although the control, non-MICs and UAs overlapped with each other to some extent (Fig. 1B1, 1B2). The supervised PLS-DA model revealed that samples from each group clustered closely and anchored away from the other groups when the samples were divided into 3 groups (Fig. 1B3). When the samples were divided into 4 groups, the majority of Mls and controls clustered separately, while the UAs and non-MICs primarily overlapped with each other, with a minority overlapping with Mls and controls (Fig. 1B4). The distant 
separation of Mls from the other groups suggested distinctly different metabolic patterns between Mls and the groups of UA and non-MICs, while the overlapping of the groups suggested similar plasma metabolic patterns between UA and the other non-MICs. In general, metabolomic data better characterized Mls than history examinations and laboratory tests, and the score plot of non-MI chest pain cases (including UA and non-MI cardiac cases) indicated that they had moderate metabolic perturbation relative to the MI cases because they anchored between $\mathrm{Ml}$ and the controls (Fig. 1B3). The above data suggest that subgroups of $\mathrm{MI}$ can be recognized by multivariate analysis of identified plasma metabolites more effectively than by routine clinical parameters.

\subsection{Pathway analysis of differential metabolites}

OPLS-DA analysis showed different metabolomic patterns of the non-Mls from the controls (Fig. 2A1). Statistical analysis suggested 50 discriminant metabolites $(p<0.05)$ that differentiated non-MI chest pain inpatients from the controls (Table 3$)$. Similarly, MI cases primarily showed different metabolomic patterns from non-MIs (Fig. 2B1). According to the statistical analysis and the VIP values, 54 discriminant metabolites were identified between Mls and non-Mls (Table 3). 
Table 3

Discriminant metabolites list: non-Mls vs controls and Mls vs non-Mls

\begin{tabular}{|c|c|c|c|c|c|c|c|c|c|c|}
\hline \multirow[t]{2}{*}{ Differential metabolites } & \multicolumn{2}{|c|}{ Controls $(n=84)$} & \multicolumn{2}{|c|}{ Ml cases $(n=85)$} & \multicolumn{2}{|c|}{ non-MI cases $(n=61)$} & \multicolumn{2}{|c|}{ MI vs non-MI } & \multicolumn{2}{|c|}{ non-MI vs Con } \\
\hline & Mean & SE & Mean & SE & Mean & SE & $\mathrm{FC}$ & T-test & FC & T-test \\
\hline Deoxyuridine & 26212 & 9533 & 1025278 & 150576 & 16840 & 555 & 60.885 & $\star \star \star$ & 0.642 & / \\
\hline Adenosine phosphosulfate & 51723 & 4141 & 99104 & 8542 & 42213 & 3793 & 2.348 & 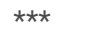 & 0.816 & / \\
\hline Deoxyadenosine monophosphate & 310296 & 7783 & 102462 & 13401 & 311056 & 7624 & 0.329 & $\star \star \star ~$ & 1.002 & / \\
\hline Guanosine diphosphate & 7379 & 469 & 27614 & 4153 & 6648 & 530 & 4.154 & 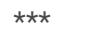 & 0.901 & / \\
\hline Inosine 2'-phosphate & 14875 & 683 & 37785 & 4687 & 14988 & 809 & 2.521 & $\star \star \star ~$ & 1.008 & / \\
\hline Adenosine monophosphate & 20124 & 1044 & 25648 & 1689 & 19902 & 1016 & 1.289 & 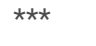 & 0.989 & / \\
\hline Hypoxanthine & 705034 & 24192 & 373454 & 29693 & 734901 & 52065 & 0.508 & $\star \star \star *$ & 1.042 & / \\
\hline Glycolate & 8319 & 247 & 9121 & 331 & 7620 & 306 & 1.197 & ** & 0.916 & / \\
\hline Methionine & 113239 & 2830 & 335994 & 27792 & 109192 & 2631 & 3.077 & 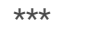 & 0.964 & / \\
\hline Arginine & 810028 & 22688 & 169566 & 34842 & 731551 & 31282 & 0.232 & $\star \star \star$ & 0.903 & / \\
\hline Valine & 2334562 & 53682 & 3710196 & 209295 & 2425637 & 63465 & 1.530 & 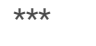 & 1.039 & / \\
\hline Citrulline & 422244 & 11079 & 296302 & 13267 & 469763 & 20870 & 0.631 & $\star \star \star$ & 1.113 & / \\
\hline Shikimate & 14871 & 796 & 8837 & 491 & 15072 & 782 & 0.586 & $\star \star \star *$ & 1.014 & / \\
\hline Ornithine & 747344 & 19685 & 522218 & 23919 & 805398 & 34957 & 0.648 & $\star \star \star *$ & 1.078 & / \\
\hline Alanine & 402892 & 10006 & 663687 & 37026 & 446350 & 16079 & 1.487 & $\star \star \star$ & 1.108 & / \\
\hline Glycine & 145111 & 2764 & 256201 & 15108 & 150041 & 3152 & 1.708 & $\star \star \star *$ & 1.034 & / \\
\hline Homocysteine & 7517 & 382 & 20169 & 2293 & 7693 & 504 & 2.622 & $\star \star \star$ & 1.023 & / \\
\hline Aspartate & 155030 & 10889 & 194713 & 12674 & 123523 & 10287 & 1.576 & 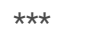 & 0.797 & / \\
\hline Ribose & 223498 & 12602 & 340299 & 15678 & 254617 & 23072 & 1.337 & $\star \star$ & 1.139 & / \\
\hline 1-Monopalmitin & 30533 & 1705 & 33208 & 1616 & 25699 & 1904 & 1.292 & ** & 0.842 & / \\
\hline Glycerate & 125449 & 4077 & 54424 & 3823 & 127894 & 10153 & 0.426 & 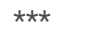 & 1.019 & / \\
\hline Citrate & 6687162 & 203004 & 1395841 & 280056 & 6186805 & 304233 & 0.226 & $\star \star \star$ & 0.925 & / \\
\hline NAD+ & 11766 & 301 & 25536 & 3262 & 11479 & 330 & 2.225 & $\star \star \star *$ & 0.976 & / \\
\hline NADPH & 5344 & 269 & 35996 & 7862 & 4157 & 192 & 8.659 & $\star \star \star$ & 0.778 & \#\#\# \\
\hline Uracil & 146594 & 7319 & 60619 & 9826 & 319956 & 18480 & 0.189 & 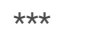 & 2.183 & \#\#\# \\
\hline Xanthine & 161018 & 5234 & 99621 & 11423 & 234923 & 11566 & 0.424 & 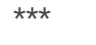 & 1.459 & \#\#\# \\
\hline Adenosine & 33452 & 2133 & 31326 & 3112 & 18676 & 1948 & 1.677 & 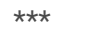 & 0.558 & \#\#\# \\
\hline IDP & 3112579 & 73422 & 1512965 & 153666 & 3597655 & 92953 & 0.421 & $\star \star \star *$ & 1.156 & \#\#\# \\
\hline Adenine & 15026 & 2519 & 13092 & 2292 & 5543 & 393 & 2.362 & ** & 0.369 & \#\#\# \\
\hline Succinate & 88550 & 1816 & 36686 & 3233 & 79439 & 2388 & 0.462 & 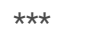 & 0.897 & $\# \#$ \\
\hline Malate & 61898 & 2263 & 35918 & 4299 & 78778 & 4163 & 0.456 & $\star \star \star$ & 1.273 & \#\#\# \\
\hline 2-Ketoglutarate & 33628 & 1314 & 21806 & 2264 & 78634 & 4864 & 0.277 & $\star \star \star ~$ & 2.338 & \#\#\# \\
\hline Acetoacetate & 132245 & 2671 & 235115 & 13087 & 160911 & 7415 & 1.461 & 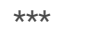 & 1.217 & \#\#\# \\
\hline Carbamoylphosphate & 29690 & 1819 & 23063 & 2179 & 36316 & 1532 & 0.635 & $\star \star \star$ & 1.223 & \#\# \\
\hline Dihydroorotate & 33819 & 1003 & 31876 & 2619 & 22310 & 579 & 1.429 & $\star \star \star *$ & 0.660 & \#\#\# \\
\hline Pantothenate & 54720 & 3022 & 38266 & 4405 & 83814 & 4827 & 0.457 & $\star \star \star$ & 1.532 & \#\#\# \\
\hline
\end{tabular}

The data was not logarized, and expressed as Mean \pm SE. Fold change (FC) were calculated by the original, non-logarized data directly. Statistical

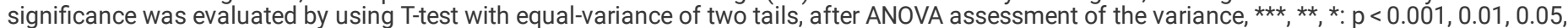
respectively, between $\mathrm{Ml}$ and non-Ml chest pain cases; \#\#\#, \#\#, \#:: p<0.001, 0.01, 0.05, respectively, between non-MI chest pain cases and the controls. 'I' represents the statistical significance of $p$ values more than 0.05 . 


\begin{tabular}{|c|c|c|c|c|c|c|c|c|c|c|}
\hline \multirow{2}{*}{$\begin{array}{l}\text { Differential metabolites } \\
\text { Phenylpyruvate }\end{array}$} & \multicolumn{2}{|c|}{ Controls $(n=84)$} & \multicolumn{2}{|c|}{ Ml cases $(n=85)$} & \multicolumn{2}{|c|}{ non-Ml cases $(n=61)$} & \multicolumn{2}{|c|}{ Ml vs non-MI } & \multicolumn{2}{|c|}{ non-Ml vs Con } \\
\hline & 51177 & 955 & 63363 & 2279 & 56537 & 1514 & 1.121 & * & 1.105 & \#\# \\
\hline Cysteine & 106041 & 3333 & 68660 & 3639 & 150632 & 7856 & 0.456 & 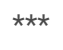 & 1.421 & \#\#\# \\
\hline Isoleucine & 1694252 & 47827 & 4794207 & 400231 & 2264556 & 105757 & 2.117 & 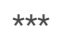 & 1.337 & \#\#\# \\
\hline Serine & 187997 & 4449 & 345414 & 14192 & 211966 & 5374 & 1.630 & 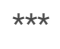 & 1.127 & \#\#\# \\
\hline Proline & 805696 & 27760 & 1281948 & 56175 & 912165 & 3390 & 1.405 & 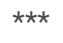 & 1.132 & \# \\
\hline Threonine & 661526 & 23707 & 1415808 & 70560 & 574241 & 19440 & 2.466 & $\star \star \star ~$ & 0.868 & \#\# \\
\hline Phenylalanine & 1051097 & 31497 & 1757945 & 133629 & 2349797 & 58409 & 0.748 & 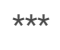 & 2.236 & \#\#\# \\
\hline Glutamine & 6299813 & 30803 & 6265669 & 37518 & 6115270 & 38706 & 1.025 & $\star \star$ & 0.971 & \#\#\# \\
\hline Histidine & 3756407 & 54216 & 2876183 & 73183 & 3370631 & 66123 & 0.853 & 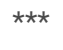 & 0.897 & \#\#\# \\
\hline Taurine & 851621 & 35791 & 1382464 & 89177 & 727822 & 36862 & 1.899 & $\star \star \star ~$ & 0.855 & $\#$ \\
\hline Lysine & 1733874 & 38073 & 505537 & 102029 & 2002743 & 55294 & 0.252 & $\star \star \star ~$ & 1.155 & \#\#\# \\
\hline N-Acetylornithine & 329453 & 18159 & 68946 & 14885 & 265119 & 11352 & 0.260 & $\star \star \star ~$ & 0.805 & $\# \#$ \\
\hline Cytosine & 7058 & 102 & 5624 & 153 & 6609 & 135 & 0.851 & 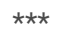 & 0.936 & $\# \#$ \\
\hline 3-Phospho-Serine & 11045 & 654 & 17870 & 1469 & 8893 & 284 & 2.009 & $\star \star \star ~$ & 0.805 & $\# \#$ \\
\hline Homoserine & 661526 & 23707 & 1415808 & 70560 & 574241 & 19440 & 2.466 & $\star \star \star ~$ & 0.868 & $\# \#$ \\
\hline 1-Monostearin & 17548 & 911 & 19128 & 896 & 13626 & 841 & 1.404 & $\star \star \star \star ~$ & 0.777 & $\# \#$ \\
\hline 2-Dehydro-D-Gluconate & 47777 & 2930 & 21494 & 3999 & 90849 & 4764 & 0.237 & $\star \star \star \star ~$ & 1.902 & \#\#\# \\
\hline Oxalate & 44511 & 2459 & 48639 & 3025 & 27020 & 2378 & 1.800 & 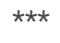 & 0.607 & \#\#\# \\
\hline Tryptophan & 647430 & 23608 & 1366119 & 100129 & 1584205 & 58633 & 0.862 & l & 2.447 & \#\#\# \\
\hline Glutamate & 253094 & 9911 & 384958 & 35729 & 384612 & 16588 & 1.001 & l & 1.520 & \#\#\# \\
\hline Hydroxyproline & 26782 & 1974 & 17148 & 1951 & 17583 & 2030 & 0.975 & / & 0.657 & \#\# \\
\hline Salicylic Acid & 4870 & 1788 & 72167 & 12248 & 57320 & 7503 & 1.259 & / & 11.771 & \#\#\# \\
\hline Pyruvate & 121954 & 5245 & 183553 & 22838 & 184240 & 7253 & 0.996 & I & 1.511 & \#\#\# \\
\hline Homocysterate & 11932 & 344 & 14822 & 1109 & 13267 & 310 & 1.117 & / & 1.112 & \#\# \\
\hline Cystathionine & 10857 & 625 & 13618 & 1322 & 17869 & 1568 & 0.762 & / & 1.646 & \#\#\# \\
\hline 2-Hydroxybutyrate & 279219 & 12481 & 421962 & 23064 & 405608 & 28986 & 1.040 & / & 1.453 & \#\#\# \\
\hline 3-Hydroxybutyrate & 52649 & 3298 & 79377 & 10282 & 85759 & 13390 & 0.926 & I & 1.629 & $\#$ \\
\hline Gluconic Acid & 105169 & 4261 & 289949 & 39667 & 203681 & 34215 & 1.424 & / & 1.937 & $\# \#$ \\
\hline Indole-3-Propanate & 55659 & 16898 & 8531 & 1748 & 5464 & 801 & 1.561 & I & 0.098 & $\# \#$ \\
\hline Glycerol & 400975 & 13803 & 290401 & 11659 & 303966 & 15733 & 0.955 & I & 0.758 & \#\#\# \\
\hline Cholesterol & 822261 & 17789 & 690461 & 14851 & 684060 & 16272 & 1.009 & I & 0.832 & \#\#\# \\
\hline Alpha-Tocopherol & 116918 & 2949 & 93627 & 2218 & 94284 & 2954 & 0.993 & / & 0.806 & \#\#\# \\
\hline Deoxyadenosine & 23123 & 1541 & 17568 & 2255 & 13041 & 1288 & 1.347 & I & 0.564 & \#\#\# \\
\hline Thymine & 544496 & 17903 & 518803 & 20345 & 457002 & 25215 & 1.135 & / & 0.839 & $\# \#$ \\
\hline Inosine & 65657 & 2868 & 28268 & 3939 & 35665 & 2829 & 0.793 & / & 0.543 & \#\#\# \\
\hline NADP+ & 24365 & 1068 & 23264 & 1803 & 19968 & 712 & 1.165 & I & 0.820 & \#\#\# \\
\hline
\end{tabular}

Of the 50 metabolites differentiating non-Mls from the controls, levels of gluconic acid and isoleucine were higher in non-Mls, while succinate, inosine, and arginine were lower, and all the above metabolites deviated further in Mls as the cardiac damage became more severe (Fig. 3C, 3D). These findings indicate that the above metabolites are involved in the development of cardiac damage. 
Although glycerol, salicylic acid, and deoxyadenosine showed significant differences between the non-MI and control groups, they had no significant difference between the $\mathrm{Ml}$ and non-MI cardiac groups. These are thus suggested as markers of non-MI chest pain. In addition to endogenous metabolites, we found that salicylic acid, an exogenous metabolite, also characterized the group of chest pain cases. Salicylic acid is the primary metabolite of aspirin, and a review of inpatient information and clinical data revealed that a large portion of chest pain patients had taken aspirin for the management of CAD.

Moreover, deviated levels of dU, methionine, homoserine, etc. were only observed in MI cases, but not between the controls and non-Mls, indicating their association with the development of MI (Fig. 3B).

A Venn diagram was created to show the discriminant metabolites between $\mathrm{MI}$ and non-Mls and those between non-Mls and controls. The overlapping region (B) in the Venn diagram (Fig. 3E) lists the metabolites screened out in both of the two comparison groups, suggesting that they were mostly likely risk factors or markers of the occurrence and development of MI, reflecting homeostatic disturbance induced by myocardia hypoxia. Figure $3 f$ shows the pathway analysis of the metabolites in the Venn $\mathrm{A}+\mathrm{B}$ region (control vs non-MI), and Fig. $3 \mathrm{G}$ shows the pathway analysis of discriminant metabolites in the Venn $\mathrm{B}+\mathrm{C}$ region (MI vs non-MI). Generally, arginine biosynthesis and pyrimidine metabolism were the most significantly altered metabolic pathways in non-MI chest pain patients' plasma compared to healthy individuals'. Enrichment and pathway analysis for the metabolites of the Venn $\mathrm{C}$ area by MetaboAnalyst showed that arginine biosynthesis $(\mathrm{p}<0.01$, FDR $<1 \%)$ was the most altered metabolic pathway (Fig. $3 \mathrm{H})$. Alanine, aspartate and glutamate metabolism $(\mathrm{p}<0.01, \mathrm{FDR}<1 \%)$ deserve attention in $\mathrm{Ml}$ as well.

\subsection{Methionine, dU and homoserine are candidate biomarkers for Ml occurrence}

A combined biosignature of homoserine, IDP and 2-ketoglutarate discriminated MI from non-MI chest pain inpatients with high accuracy (Fig. 3L,AUC = 0.98, sensitivity: $94.1 \%$, specificity: $100 \%)$.

The potential capacity of each discriminant metabolite to diagnose MI was assessed by ROC analysis (Table 4). Notably, although pathway analysis did not draw our attention to the methionine-related metabolic module, methionine and homoserine showed their potential in distinguishing MI from non-MI cardiac cases. Homoserine (AUC $=0.94$; specificity $=100 \%$, sensitivity $=81 \%$ ) was more specific for MI diagnosis but less sensitive than methionine (AUC $=0.96$; specificity $=94.6 \%$; sensitivity $=89.4 \%$ ) (Fig. $3 \mathrm{~J})$. dU also scored highly, with an AUC over $90 \%$ (Fig. 3F). Some other metabolites that showed good diagnostic potential were cysteine, 2-ketoglutarate, IDP, and uracil (Table 4).

Table 4

Differential metabolites and the diagnostic potential between $\mathrm{Ml}$ and non-MI chest pain cases

\begin{tabular}{|llllllc|}
\hline Differential metabolites & AUROC & 95\%C.I. & Sensitivity & Specificity & log(OR) & $95 \%$ C.I. \\
\hline Homoserine\&IDP\&a-Ketoglutarate & 0.9810 & $0.9614-1.0000$ & $94.10 \%$ & $100 \%$ & 3.02 & $2.12-3.93$ \\
\hline Methionine & 0.9643 & $0.9309-0.9978$ & $89.40 \%$ & $94.60 \%$ & 3.48 & $2.01-4.94$ \\
\hline Homoserine & 0.9431 & $0.893-0.9932$ & $80.90 \%$ & $100 \%$ & 1.61 & $1.04-2.18$ \\
\hline a-Ketoglutarate & 0.9390 & $0.8876-0.9905$ & $100 \%$ & $0.00 \%$ & -0.11 & $-0.28--0.03$ \\
\hline Uracil & 0.9166 & $0.8585-0.9747$ & $100 \%$ & $0.00 \%$ & -2.38 & $-3.45--1.32$ \\
\hline Deoxyuridine & 0.9166 & $0.8462-0.987$ & $80.90 \%$ & $100 \%$ & 3.01 & $1.71-4.30$ \\
\hline 2-Dehydro-D-gluconate & 0.9040 & $0.8362-0.9717$ & $2.10 \%$ & $100 \%$ & -1.69 & $-2.53--0.84$ \\
\hline Cysteine & 0.8976 & $0.8302-0.9651$ & $100 \%$ & $0.00 \%$ & -0.41 & $-0.61--0.20$ \\
\hline Deoxyadenosine monophosphate & 0.8976 & $0.8335-0.9618$ & $100 \%$ & $0.00 \%$ & -5.57 & $-8.61--2.53$ \\
\hline IDP & 0.8838 & $0.8124-0.9553$ & $100 \%$ & $0.00 \%$ & -5.15 & $-7.86--2.44$ \\
\hline Glyceric acid & 0.8758 & $0.8022-0.9493$ & $100 \%$ & $0.00 \%$ & -1.53 & $-2.08--0.98$ \\
\hline Citrate & 0.8568 & $0.7774-0.9362$ & $100 \%$ & $0.00 \%$ & -1.69 & $-2.50--0.88$ \\
\hline Succinate & 0.8562 & $0.7768-0.9357$ & $100 \%$ & $0.00 \%$ & -1.48 & $-2.14--0.83$ \\
\hline Pantothenate & 0.8332 & $0.7452-0.9213$ & $100 \%$ & $0.00 \%$ & -0.98 & $-1.36--0.59$ \\
\hline Xanthine & 0.8240 & $0.7312-0.9168$ & $2.10 \%$ & $100 \%$ & -1.06 & $-1.46--0.65$ \\
\hline Arginine & 0.8235 & $0.7319-0.9151$ & $2.10 \%$ & $100 \%$ & -0.82 & $-1.10--0.53$ \\
\hline Logarization transformation of the data was applied to the data before the calculation. & & & \\
\hline
\end{tabular}

Moreover, to assess the role of these metabolites as risk factors for the prediction of MI occurrence, OR values were calculated between the MI and nonMI groups. Homoserine, dU and methionine had high scores (Table 4). After adjusting for age, sex, LDL-c, HDL-c, smoking/diabetic/hypertensive history, LogOR of methionine, homoserine and dU, all had ORs greater than 1 (Mls vs non-Mls. Figure 3I). Additionally, the ORs of succinate, IDP and glyceric acid were much less than 1, indicating they are potential protective factors for MI (Table 4). 


\subsection{Traditional CAD risk factors and cardiac function influence the metabolic pattern}

Correlation analysis showed that methionine, homoserine, homocysteine and dU were all affected by smoking history, but none was obviously perturbed by hypertension (Table S3). However, in the subgroup analyses of smoking/nonsmoking, hypertensive/normotensive, diabetic/nondiabetic, aged 4554/55-65 and male/female, means of homoserine, methionine and dU were higher in Ml cases (Table 5).

Table 5

Mean of deoxyuridine, methionine and homoserine in certain subgroups of the controls, non-Mls and MI cases

\begin{tabular}{|c|c|c|c|c|c|c|c|c|c|}
\hline Risk factors & $\begin{array}{l}\text { Sub- } \\
\text { groups }\end{array}$ & Deoxyurdine & Methionine & Homoserine & $\begin{array}{l}\text { non-Risk } \\
\text { factors }\end{array}$ & $\begin{array}{l}\text { Sub- } \\
\text { groups }\end{array}$ & Deoxyurdine & Methionine & Homoserine \\
\hline \multirow[t]{3}{*}{ Smokers } & $\begin{array}{l}\text { The } \\
\text { controls }\end{array}$ & 4.22 & 5.04 & 5.82 & \multirow[t]{3}{*}{ non Smokers } & $\begin{array}{l}\text { The } \\
\text { controls }\end{array}$ & 4.24 & 5.05 & 5.8 \\
\hline & $\begin{array}{l}\text { non-MI } \\
\text { cases }\end{array}$ & 4.22 & 5.03 & 5.76 & & $\begin{array}{l}\text { non-MI } \\
\text { cases }\end{array}$ & 4.22 & 5.03 & 5.75 \\
\hline & $\begin{array}{l}\text { Ml } \\
\text { cases }\end{array}$ & 5.48 * \# & 5.44 * \# & 6.13 *\# & & $\begin{array}{l}\text { Ml } \\
\text { cases }\end{array}$ & $5.45^{*}$ * & $5.43 *$ * \# & 6.11 *\# \\
\hline \multirow[t]{3}{*}{ Diabetic } & $\begin{array}{l}\text { The } \\
\text { controls }\end{array}$ & 4.22 & 5.04 & 5.8 & \multirow[t]{3}{*}{$\begin{array}{l}\text { non-diabetic } \\
\text { or insulin } \\
\text { resistance }\end{array}$} & $\begin{array}{l}\text { The } \\
\text { controls }\end{array}$ & 4.24 & 5.05 & 5.8 \\
\hline & $\begin{array}{l}\text { non-MI } \\
\text { cases }\end{array}$ & 4.2 & 5.03 & 5.75 & & $\begin{array}{l}\text { non-MI } \\
\text { cases }\end{array}$ & 4.21 & 5.04 & 5.73 \\
\hline & $\begin{array}{l}\text { MI } \\
\text { cases }\end{array}$ & 5.44 * \# & $5.43 * \#$ & 6.12 *\# & & $\begin{array}{l}\text { MI } \\
\text { cases }\end{array}$ & 5.34 * \# & $5.40 * \#$ & $6.09 * \#$ \\
\hline \multirow[t]{3}{*}{ Hypertensive } & $\begin{array}{l}\text { The } \\
\text { controls }\end{array}$ & 4.24 & 5.05 & 5.8 & \multirow[t]{3}{*}{ Normotensive } & $\begin{array}{l}\text { The } \\
\text { controls }\end{array}$ & 4.22 & 5.04 & 5.8 \\
\hline & $\begin{array}{l}\text { non-MI } \\
\text { cases }\end{array}$ & 4.22 & 5.03 & 5.75 & & $\begin{array}{l}\text { non-MI } \\
\text { cases }\end{array}$ & 4.22 & 5.04 & 5.73 \\
\hline & $\begin{array}{l}\text { Ml } \\
\text { cases }\end{array}$ & $5.40 *$ * \# & 5.42 * \# & 6.11 *\# & & $\begin{array}{l}\text { Ml } \\
\text { cases }\end{array}$ & 5.37 * \# & 5.42 * \# & $6.10 * \#$ \\
\hline \multirow[t]{3}{*}{$\begin{array}{l}\text { Age of 45- } \\
54\end{array}$} & $\begin{array}{l}\text { The } \\
\text { controls }\end{array}$ & 4.29 & 5.07 & 5.83 & \multirow[t]{3}{*}{ Age: 55-65 } & $\begin{array}{l}\text { The } \\
\text { controls }\end{array}$ & 4.23 & 5.03 & 5.83 \\
\hline & $\begin{array}{l}\text { non-MI } \\
\text { cases }\end{array}$ & 4.21 & 5.05 & 5.72 & & $\begin{array}{l}\text { non-MI } \\
\text { cases }\end{array}$ & 4.18 & 5.04 & 5.75 \\
\hline & $\begin{array}{l}\text { Ml } \\
\text { cases }\end{array}$ & 5.30 * \# & $5.37 *$ * \# & $6.13^{*, \#}$ & & $\begin{array}{l}\text { MI } \\
\text { cases }\end{array}$ & 5.60 * \# & 5.45 * \# & 6.11 *\# \\
\hline \multirow[t]{3}{*}{ Male } & $\begin{array}{l}\text { The } \\
\text { controls }\end{array}$ & 4.22 & 5.05 & 5.8 & \multirow[t]{3}{*}{ Female } & $\begin{array}{l}\text { The } \\
\text { controls }\end{array}$ & 4.28 & 5.05 & 5.82 \\
\hline & $\begin{array}{l}\text { non-MI } \\
\text { cases }\end{array}$ & 4.22 & 5.05 & 5.77 & & $\begin{array}{l}\text { non-MI } \\
\text { cases }\end{array}$ & 4.23 & 5 & 5.72 \\
\hline & $\begin{array}{l}\text { MI } \\
\text { cases }\end{array}$ & $5.43 * \#$ & 5.44 * \# & 6.11 * & & $\begin{array}{l}\text { MI } \\
\text { cases }\end{array}$ & 5.32 *\# & $5.35^{*} \#$ & $6.10 * \#$ \\
\hline
\end{tabular}

* MI vs controls, $\mathrm{p}<0.05$; \#, MI vs non-MI $\mathrm{p}<0.05$.

All data were logarized before calculating, the result showed means of each subgroup.

As a clinical indicator of cardiac function in MI, positive NT-proBNP represents cardiac dysfunction. Methionine, homoserine and deoxyuridine were further elevated in NT-proBNP-positive cases. Pathway analysis of the discriminant metabolites (Table S4) between the NT-proBNP positive and negative groups suggested that only arginine biosynthesis was severely impaired $(P<0.001, F D R<1 \%)$, indicating that arginine biosynthesis is closely associated with cardiac function. (Figure S3A)

cTnt, CK-MB, AST, LDH and HBDH are well-recognized indicators involved in myocardial damage and infarction. Six metabolites, 2-hydroxybutyrate, 3hydroxybutyrate, homocysteine, palmitic acid, stearic acid and 1-monooleoylglycerol, were positively and significantly correlated with both cTNT and CKMB (Figure S3C). As candidate predictors of MI, methionine, dU and homoserine were significantly and positively correlated with LDH, HBDH and AST (Figure S3B).

\subsection{Altered plasma pyrimidine and methionine metabolism in MI cases}

The pathway of pyrimidine metabolism was deranged in the MI cases, as shown by the dramatic changes in dU and uracil. Figure 4D shows metabolites and metabolic enzymes in the dU-related pathway. According to a circulating transcriptomics dataset (GEO accession: GSE48060), the dU-related enzymes cytidine deaminase (CDA) and uridine phosphorylase 1 (UPP1) are upregulated in MI patient plasma. According to The Human Protein Atlas, 
CDA and UPP1 are highly enriched in immune cells (mainly in neutrophils and monocytes). Figure 4G shows white blood cell (WBC) counts, neutrophil (NE) counts, monocyte (MO) and lymphocyte (LY) counts and dU abundance in MI and non-MI cases. Immune cells generally increased in MI plasma. However, statistically, the correlation analysis did not detect a relationship between dU abundance and the number of any type of immune cell (including total WBCs).

Our study on transverse aortic constriction (TAC) mouse models showed that cardiac CDA mRNA expression increased as BNP and ANF mRNA levels increased (Figure S3 D-F,H). dU level is significantly higher in the proBNP-positive group than in the proBNP-negative chest pain group (Fig. 4H). We hypothesize that $\mathrm{dU}$ is also a potential cardiac function marker.

Figure 4A shows methionine, homocysteine and cystathionine in a panel. Inconsistent with the observed elevation of methionine, circulating transcriptomics of MI patients (GEO accession: GSE48060) showed that among the methionine abundance-related genes, 5-MethyltetrahydrofolateHomocysteine Methyltransferase (MTR) expression decreased and Cystathionine $\beta$-synthase (CBS) increased ( $p<0.05$, Fig. 4B). The other genes involved in methionine turnover, including methionine-tRNA ligase (MetRS), MrsB, MrsA (MARS), Mat2a and Mat1a, remained statistically unchanged in plasma. The above analysis from circulating transcriptomics suggested that cells in plasma are not likely to be responsible for the marked elevation of methionine in MI patients' blood. Instead, it is more likely that damaged cardiac tissues (or other tissues) release more or utilize less free methionine. This hypothesis is supported by the fact that a transcriptomics study of a MI mouse model (LAD) revealed that in ischemic cardiac tissue, MetRS decreased significantly in MI mouse cardiac samples. (Fig. 4C; GEO accession: GSM12346)

\section{Discussions}

The onset of myocardial infarction is always urgent, sudden and sometimes associated with a dim prognosis. Early warning and recognition of myocardial infarction is a great challenge for clinicians and is of crucial importance to treat suspected MI patients. The early detection and intervention of $\mathrm{MI}$ require growing knowledge of the biology data of $\mathrm{MI}$ patients. In this study, we demonstrated that metabolomic data well discriminate $\mathrm{MI}$ cases from other non-MI chest pain cases. Some metabolites have the potential ability to differentiate and diagnose MI patients among CAD patients with the symptom of chest pain and suggest potential markers to predict the onset of $\mathrm{Ml}$.

\subsection{Plasma metabolic features and metabolite biomarkers for the diagnosis of MI}

This study identified a panel of discriminant metabolites that were also suggested as potential markers of MI in previous reports, such as taurine, methionine, leucine, isoleucine, valine, ornithine, tryptophan, citrate, and 2-ketoglutarate.[21] To our surprise, some of the metabolites showed great potential in the diagnosis of MI. Both elevated and decreased levels of metabolites were observed in the Ml group. Among the differential metabolites between $\mathrm{Ml}$ cases and non-MI cases, 10 of them in the Ml group had twice the abundance as in the non-MI group (Mls/non-Mls, FC > 2); 17 metabolites had less than half the abundance as in the non-MI cases (MIs/non-MIs, FC $<0.5)$.

\section{Elevated metabolites in Ml cases}

Based on metabolites that were elevated in the Ml group, we found that methionine and cysteine metabolisms are involved in MI. In this metabolic module, methionine is a precursor of homocysteine, homoserine is utilized in the biosynthesis of methionine, and homoserine was also reported as a serum marker for cardiac disease in atherosclerosis patients with stent restenosis [22].

Although the causes of plasma metabolite alterations could be many (e.g., gut microbial metabolites), we evaluated plasma transcriptomics data because circulating substances influence plasma metabolites most directly. Transcriptomics data showed that plasma methionine-related mRNA tends to utilize methionine and homocysteine to produce more cystathionine. Yet there was not a significant change of the key genes involved in the pathway of methionine transsulfuration. Plasma Cystathionine- $\beta$-synthase (CBS) and cystathionine-y-lyase (CTH) a similar rna level between control and Mls, indicating blood cells methionine transsulfuration pathway in mRNA level cannot explain the elevation of methionine. One explanation is hinted at by murine MI models showing decreased levels of MARS mRNA in the heart, which suggests that the damaged heart has difficulty binding methionine to tRNA. As a result, free methionine is released into the blood and detected in the circulatory system of MI patients.

The pyrimidine metabolism pathway was also deranged in the MI cases. The elevation of dU has been identified as a potential adverse factor for nucleotide pool balance and mitochondrial function in the case of mitochondrial neurogastrointestinal encephalomyopathy[23, 24]. As a metabolite in pyridine metabolism, dU has never been suggested to play a role in cardiovascular diseases before. Unlike methionine, the elevation of dU is more likely to be related to blood cells. A previous study[25] (Dateset: GSE103182) showed that STEMI (ST-elevation myocardial infarction) patients express more neutrophil-specific CDA mRNA in plasma than NSTEMI (non-ST-elevation myocardial infarction) patients. Consistent with this finding, our data showed that in the STEMI group, both dU and neutrophil counts were higher than those in the NSTEMI group (Figure S3G). It is possible that granulocyte-specific CDA is enriched in one subset of neutrophils. The subset of neutrophils varies significantly among non-MI, STEMI and NSTEMI cases. Alternatively, the elevation of CDA could be due to the heightened necrosis of granulocytes.

\section{Decreased metabolites in Ml cases}


Among all the discriminant metabolites that declined in MI cases, arginine was the most noteworthy (Fig. $3 \mathrm{C}$ ). ROC analysis showed arginine AUC equals to 0.8865 (Mls vs non-MIs). Our study showed that as cardiac damage and function worsened, these plasma arginine biosynthesis-related metabolites dropped more (Table 3). However, arginine's role as a CAD risk factor may be underestimated because arginine bioavailability, not just arginine abundance, was more significantly changed in MI patients. Another study reported that diminished global arginine bioavailability is predictive of increased CAD risk [16].

Uracil, a pyrimidine found in RNA, was also significantly decreased in MI plasma. Figure 4D and 4F show the relationship of uracil and dU in plasma pyrimidine metabolism. The panel indicates the elevated demand for plasma uracil in $\mathrm{Ml}$ cases and the enhanced demand for damage repair after heart attack. This suggests that Ml occurrence may lead to the body continues to repair itself by producing more RNA or even DNA[26].

\subsection{Potential markers for predicting the occurrence of MI}

\section{Traditional risk factors}

As indicated by adjusted and unadjusted OR values, traditional risk factors, such as diabetes, hypertension, and smoking, had confounding effects on the candidate metabolite biomarkers[27]. In this study, diabetes and smoking also ranked the marked risk factors for MI occurrence, but the history of hypertension was not $(\mathrm{OR}<1, \mathrm{p}>0.05)$. As for the key substances in lipid metabolism, higher levels of TC, TG and LDLc were confirmed as risk factors for MI groups (Mls vs non-MIs, Table S5). However, higher level of LDLc failed to demonstrate itself as a "risk" to develop non-MI cardiac chest pain. (non-Mls vs the control, Table S5). Considering that routine therapy with anti-lipidemic and antihypertensive drugs lowered LDLC and blood pressure levels, medications could be responsible for some of the controversial findings.

\section{Metabolite risk factors}

The panel of discriminant metabolites did not only offer metabolic markers facilitating the diagnosis of MI, but also contributed potential markers suggesting the conceivable occurrence of $\mathrm{MI}$ for prediction. Previous studies reported that methionine and dU can be promotors for CAD development because their accumulation may lead to more adverse effects on humans, e.g., high levels of methionine have been identified as atherogenic[28] and metabotoxic[29] for a long time, while dU acts as a conventional antimetabolite[30]. For the first time, our data suggest that methionine and dU are highrisk markers for predicting patients about the occurrence of MI. Moreover, consistent with a previous report suggesting the relationship of dU with insulin resistance[31], our correlation analysis also suggested that $\mathrm{dU}$ is partially affected by diabetes (correlation analysis vs diabetes, $p<0.05$, Pearson's $r=$ 0.23), in addition to tobacco use and HDLc level.

As a precursor of homocysteine, methionine elevation is supposed to be a promoter for the development of CAD[32]. Consistent with that idea, a previous study showed that methionine in CAD cases is significantly higher than in healthy controls and is a risk factor for CAD occurrence in an unadjusted model[14]. However, after adjusting for race, sex, age, diabetes, smoking and hypertension, methionine is not as dangerous as it is in an unadjusted model [33]. Moreover, our data showed that methionine was an independent biomarker for MI. Notably, we found that the diagnostic performance of biomarker candidates for MI varied with individual characteristics, such as diabetis. When we studied individuals with a history of diabetes, methionine achieved an AUC score as high as 100\% (sensitivity: 100.00\%, specificity: $100.00 \%$ ). For nondiabetic inpatients, the AUC score of methionine was only $73 \%$, which is much lower than that of diabetic inpatients. Generally, this study suggests some metabolites that have the potential to facilitate the diagnosis and prediction of MI. We believe that the traditional marker (such as Tnt) and the metabolic markers (such as dU and methionine) reflect the damage of heart at different angles that are involved in confounding factors contributing to the onset of MI. In line with most studies, the odds ratio values consistently suggested that LDL cholesterol levels is the traditional common risk factor for cardiovascular disease [34]. Unexpectedly, in this study, a high level of LDLc (whether or not in an abnormal range) is not a risk for cardiac chest pain patients. Similarly, hypertension is a risk for developing CAD-related chest pain by healthy people, but not for the development of MI by chest pain patients. It greatly challenges our further research of the underlying mechanism.

\section{Conclusion}

Plasma deoxyuridine, homoserine, methionine, as well as a combination of homoserine, IDP and 2-ketoglutarate were suggested as markers for MI diagnosis. The changes of some metabolites abundance in diseased samples can be explained by plasma transcriptomics and could be highly associated with the blood cells. Hopefully, the growing plasma single-cell RNA sequencing evidence [35, 36] and omics data (e.g., cfDNA methylome[37]) offer a clue to reveal the mechanism behind the changes soon.

\section{Abbreviations}

MI, myocardial infarction; CAD, coronary artery disease; UA, unstable angina; non-MIs, non-MI chest pain cases; ECG, Electrocardiogram; ROC, receiver operating characteristic curve; AUC, area under the ROC Curve; QC, quality control; IS, internal standard; VIP, variable Importance; QC , quality control, IS, internal standard, PCA, Principal component analysis, PLS-DA, partial least square to latent structure discriminant analysis; OPLS-DA, orthogonal PLSDA; OR, odds ratios; dU, deoxyuridine; TAC, transverse aortic constriction; LAD, left anterior descending coronary artery; CDA, cytidine deaminase; UPP1, uridine phosphorylase 1; MARS, methionyl-tRNA synthetase; WBC, white blood cell counts; NE, neutrophil; MO, monocyte and LY, lymphocyte.

Page 13/19 


\section{Declarations}

\subsection{Ethics approval and consent to participate}

The study followed the principles outlined in the Declaration of Helsinki, informed written consent was given prior to the inclusion of subjects in the study. The study was also under the guidance and supervision of Ethics Committee of the First Affiliated Hospital of Nanjing Medical University (Lot number: 2018-SR-028).

\subsection{Consent for publication}

Not applicable.

\subsection{Availability of data and materials}

The raw data analyzed in the study are available from the corresponding author on reasonable request.

\subsection{Competing interests}

The authors declare that they have no competing interests.

\subsection{Funding}

This work was supported by the National Key Special Project of Science and Technology for Innovation Drugs of China (2017ZX09301013), the leading technology foundation research project of Jiangsu province (BK20192005).

\subsection{Authors' contributions}

Prof. Xiangqing Kong, Jiye Aa came up with the idea of the study; Prof. Guangji Wang and Jiye Aa provided the LC/MS and GC/MS platform. Dr. Zhijian Yang, Liansheng Wang and Chunjian Li confirmed the diagnosis of MI, non-MI chest pain cases. Ms. Nan Aa, Ying Lu and Heng Tang collected the blood samples, recorded the medical history of the volunteers and prepared the plasma samples. Ms. Nan Aa, Mr. Zhenyao Lu and Dr. Runbing Sun performed the untargeted metabolomics. Ms. Nan Aa and Mr. Mengjie Yu analyzed the data. Figures and tables were produced by Ms. Nan Aa, Mr. Mengjie Yu and Prof. Jiye Aa. Prof. Xiangqing Kong, Ms. Nan Aa and Ms. Ying Lu wrote the manuscript.

\subsection{Acknowledgements}

We thank Mrs. Dongmei Shi and all nursing staffs in department of cardiology for the guidance and assistance in collection of the blood samples. We thank Prof. Yongyue Wei, School of public health, Nanjing Medical University, for his valuable suggestion on the statistics.

\section{References}

1. Hammarsten O, Mair J, Mockel M, Lindahl B, Jaffe AS. Possible mechanisms behind cardiac troponin elevations. Biomarkers. 2018;23:725-34.

2. Nowak R, Mueller C, Giannitsis E, Christ M, Ordonez-Llanos J, DeFilippi C, McCord J, Body R, Panteghini M, Jernberg T, Plebani M, Verschuren F, French JK, Christenson R, Jacobsen G, Dinkel C, Lindahl B. High sensitivity cardiac troponin T in patients not having an acute coronary syndrome: results from the TRAPID-AMI study. Biomarkers. 2017;22:709-14.

3. Riedlinger D, Mockel M, Muller C, Holert F, Searle J, von Recum J, Slagman A. High-sensitivity cardiac troponin T for diagnosis of NSTEMI in the elderly emergency department patient: a clinical cohort study. Biomarkers. 2018;23:551-7.

4. Lopaschuk GD. Metabolic Modulators in Heart Disease: Past, Present, and Future. Can J Cardiol. 2017;33:838-49.

5. Brindle JT, Antti H, Holmes E, Tranter G, Nicholson JK, Bethell HW, Clarke S, Schofield PM, McKilligin E, Mosedale DE, Grainger DJ. Rapid and noninvasive diagnosis of the presence and severity of coronary heart disease using 1H-NMR-based metabonomics. Nat Med. 2002;8:1439-44.

6. Sabatine MS, Liu E, Morrow DA, Heller E, McCarroll R, Wiegand R, Berriz GF, Roth FP, Gerszten RE. Metabolomic identification of novel biomarkers of myocardial ischemia. Circulation. 2005;112:3868-75.

7. Shah SH, Kraus WE, Newgard CB. Metabolomic profiling for the identification of novel biomarkers and mechanisms related to common cardiovascular diseases: form and function. Circulation. 2012;126:1110-20.

8. Lewis GD, Asnani A, Gerszten RE. Application of metabolomics to cardiovascular biomarker and pathway discovery. J Am Coll Cardiol. 2008;52:117-23. 
9. Fan Y, Li Y, Chen Y, Zhao YJ, Liu LW, Li J, Wang SL, Alolga RN, Yin Y, Wang XM, Zhao DS, Shen JH, Meng FQ, Zhou X, Xu H, He GP, Lai MD, Li P, Zhu W, Qi LW. Comprehensive Metabolomic Characterization of Coronary Artery Diseases. J Am Coll Cardiol. 2016;68:1281-93.

10. Cheng ML, Wang CH, Shiao MS, Liu MH, Huang YY, Huang CY, Mao CT, Lin JF, Ho HY, Yang NI. Metabolic disturbances identified in plasma are associated with outcomes in patients with heart failure: diagnostic and prognostic value of metabolomics. J Am Coll Cardiol. 2015;65:1509-20.

11. Stegemann C, Pechlaner R, Willeit P, Langley SR, Mangino M, Mayr U, Menni C, Moayyeri A, Santer P, Rungger G, Spector TD, Willeit J, KiechI S, Mayr M. Lipidomics profiling and risk of cardiovascular disease in the prospective population-based Bruneck study. Circulation. 2014;129:1821-31.

12. Zhang L, Wei TT, Li Y, Li J, Fan Y, Huang FQ, Cai YY, Ma G, Liu JF, Chen QQ, Wang SL, Li H, Alolga RN, Liu B, Zhao DS, Shen JH, Wang XM, Zhu W, Li P, Qi LW, Functional Metabolomics Characterizes a Key Role for N-Acetylneuraminic Acid in Coronary Artery Diseases, Circulation, 2018, 137: 13741390.

13. Song JP, Chen L, Chen X, Ren J, Zhang NN, Tirasawasdichai T, Hu ZL, Hua W, Hu YR, Tang HR, Chen HV, Hu SS. Elevated plasma betahydroxybutyrate predicts adverse outcomes and disease progression in patients with arrhythmogenic cardiomyopathy, Sci Transl Med, $2020,12$.

14. Wald DS, Law M, Morris JK. Homocysteine and cardiovascular disease: evidence on causality from a meta-analysis. BMJ. 2002;325:1202.

15. Floegel A, Kuhn T, Sookthai D, Johnson T, Prehn C, Rolle-Kampczyk U, Otto W, Weikert C, Illig T, von Bergen M, Adamski J, Boeing H, Kaaks R, Pischon T. Serum metabolites and risk of myocardial infarction and ischemic stroke: a targeted metabolomic approach in two German prospective cohorts. Eur J Epidemiol. 2018;33:55-66.

16. Tang WH, Wang Z, Cho L, Brennan DM, Hazen SL. Diminished global arginine bioavailability and increased arginine catabolism as metabolic profile of increased cardiovascular risk. J Am Coll Cardiol. 2009;53:2061-7.

17. Grund B, Sabin C. Analysis of biomarker data: logs, odds ratios, and receiver operating characteristic curves. Curr Opin HIV AIDS. 2010;5:473-9.

18. Trygg JA,J, Gullberg J, Johansson Al, Jonsson P, Antti H, Marklund SL, Moritz T. Extraction and GC/MS analysis of the human blood plasma metabolome. Anal Chem. 2005;77:8086-94.

19. Aa N, Guo JH, Cao B, Sun RB, Ma XH, Chu Y, Zhou SP, Aa JY, Yang ZJ, Sun H, Wang GJ. Compound danshen dripping pills normalize a reprogrammed metabolism of myocardial ischemia rats to interpret its time-dependent efficacy in clinic trials: a metabolomic study. Metabolomics. 2019;15:128.

20. Zhang Y, Lu W, Wang Z, Zhang R, Xie Y, Guo S, Jiao L, Hong Y, Di Z, Wang G, Aa J. Reduced Neuronal cAMP in the Nucleus Accumbens Damages Blood-Brain Barrier Integrity and Promotes Stress Vulnerability. Biol Psychiatry. 2020;87:526-37.

21. Sansbury BE, DeMartino AM, Xie Z, Brooks AC, Brainard RE, Watson LJ, DeFilippis AP, Cummins TD, Harbeson MA, Brittian KR, Prabhu SD, Bhatnagar A, Jones SP, Hill BG. Metabolomic analysis of pressure-overloaded and infarcted mouse hearts. Circ Heart Fail. 2014;7:634-42.

22. Hasokawa M, Shinohara M, Tsugawa H, Bamba T, Fukusaki E, Nishiumi S, Nishimura K, Yoshida M, Ishida T, Hirata K. Identification of biomarkers of stent restenosis with serum metabolomic profiling using gas chromatography/mass spectrometry. Circ J. 2012;76:1864-73.

23. Bax BE, Levene M, Bain MD, Fairbanks LD, Filosto M, Kalkan Ucar S, Klopstock T, Kornblum C, Mandel H, Rahman S, Roubertie A, Scarpelli M, Sedgwick PM, Baru M, Sellos-Moura M, Price J, Horn P, Nirmalananthan N. Erythrocyte Encapsulated Thymidine Phosphorylase for the Treatment of Patients with Mitochondrial Neurogastrointestinal Encephalomyopathy: Study Protocol for a Multi-Centre, Multiple Dose, Open Label Trial, J Clin Med, 2019, 8.

24. Marti R, Nishigaki Y, Hirano M. Elevated plasma deoxyuridine in patients with thymidine phosphorylase deficiency. Biochem Biophys Res Commun. 2003;303:14-8.

25. Chiesa M, Piacentini L, Bono E, Milazzo V, Campodonico J, Marenzi G, Colombo GI. Whole blood transcriptome profile at hospital admission discriminates between patients with ST-segment elevation and non-ST-segment elevation acute myocardial infarction. Sci Rep. 2020;10:8731.

26. Krokan HE, Drablos F, Slupphaug G. Uracil in DNA-occurrence, consequences and repair. Oncogene. 2002;21:8935-48.

27. Foody J, Huo Y, Ji L, Zhao D, Boyd D, Meng HJ, Shiff S, Hu D. Unique and Varied Contributions of Traditional CVD Risk Factors: A Systematic Literature Review of CAD Risk Factors in China. Clin Med Insights Cardiol. 2013;7:59-86.

28. Troen AM, Lutgens E, Smith DE, Rosenberg IH, Selhub J. The atherogenic effect of excess methionine intake. Proc Natl Acad Sci U S A. 2003;100:15089-94.

29. Murphy-Chutorian DR, Wexman MP, Grieco AJ, Heininger JA, Glassman E, Gaull GE, Ng SK, Feit F, Wexman K, Fox AC. Methionine intolerance: a possible risk factor for coronary artery disease. J Am Coll Cardiol. 1985;6:725-30.

30. Das KC, Herbert V. The lymphocyte as a marker of past nutritional status: persistence of abnormal lymphocyte deoxyuridine (dU) suppression test and chromosomes in patients with past deficiency of folate and vitamin B12. Br J Haematol. 1978;38:219-33.

31. Li LO, Hu YF, Wang L, Mitchell M, Berger A, Coleman RA. Early hepatic insulin resistance in mice: a metabolomics analysis. Mol Endocrinol. 2010;24:657-66.

32. Kim J, Kim H, Roh H, Kwon Y. Causes of hyperhomocysteinemia and its pathological significance. Arch Pharm Res. 2018;41:372-83.

33. Shah SH, Bain JR, Muehlbauer MJ, Stevens RD, Crosslin DR, Haynes C, Dungan J, Newby LK, Hauser ER, Ginsburg GS, Newgard CB, Kraus WE. Association of a peripheral blood metabolic profile with coronary artery disease and risk of subsequent cardiovascular events. Circ Cardiovasc Genet. 2010;3:207-14.

34. Holmes MV, Millwood IY, Kartsonaki C, Hill MR, Bennett DA, Boxall R, Guo Y, Xu X, Bian Z, Hu R, Walters RG, Chen J, Ala-Korpela M, Parish S, Clarke RJ, Peto R, Collins R, Li L, Chen Z. G. China Kadoorie Biobank Collaborative, Lipids, Lipoproteins, and Metabolites and Risk of Myocardial Infarction 
and Stroke. J Am Coll Cardiol. 2018;71:620-32.

35. Abplanalp WT, John D, Cremer S, Assmus B, Dorsheimer L, Hoffmann J, Becker-Pergola G, Rieger MA, Zeiher AM, Vasa-Nicotera M, Dimmeler S. Single cell RNA sequencing reveals profound changes in circulating immune cells in patients with heart failure, Cardiovasc Res, 2020.

36. Ren H, Liu X, Wang L, Gao Y. Lymphocyte-to-Monocyte Ratio: A Novel Predictor of the Prognosis of Acute Ischemic Stroke. J Stroke Cerebrovasc Dis. 2017;26:2595-602.

37. Zemmour H, Planer D, Magenheim J, Moss J, Neiman D, Gilon D, Korach A, Glaser B, Shemer R, Landesberg G, Dor Y. Non-invasive detection of human cardiomyocyte death using methylation patterns of circulating DNA. Nat Commun. 2018;9:1443.

\section{Figures}
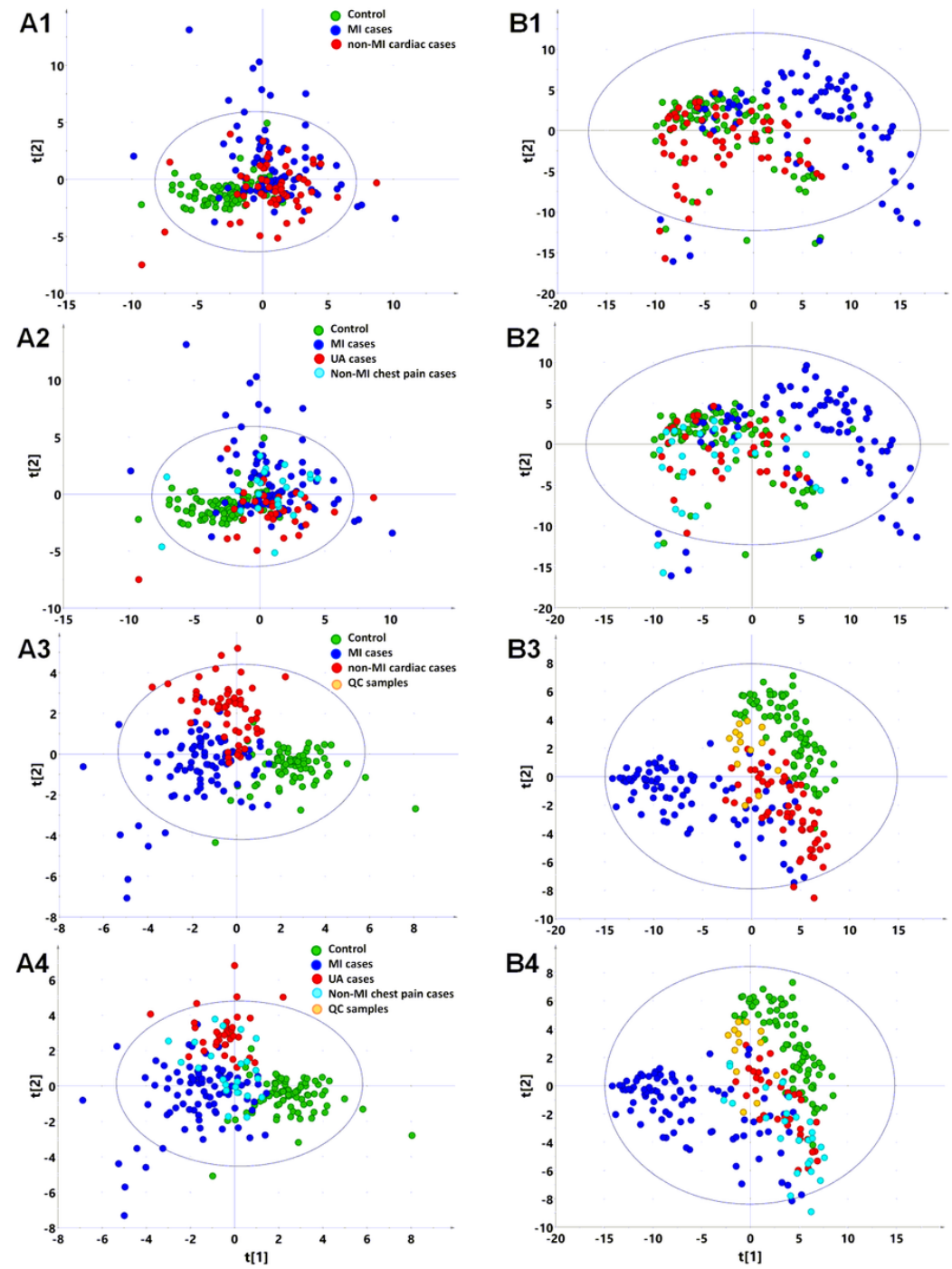

Figure 1

Multivariate statistical analysis differentiates the groups of $\mathrm{Ml}$ cases, non-MI chest pain cases and the controls based on clinical information(A) and metabolomic data(B), respectively. 1, Non-supervised PCA modeling displays the original similarity of three groups of MI cases(n=85), non-MI chest pain cases $(n=61)$ and the controls $(n=84)$, without arbitrary grouping. 2, Non-supervised PCA modeling displays the original similarity of four groups of MI cases, UA, other non-MI cardiac cases and the controls, without arbitrary grouping. A1\&A2, PC1:R2X(cum) =0.0895, Q2(cum)=0.0106; PC2:R2X(cum) =0.167, Q2(cum)=0.0356. B1\&B2, PC1:R2X(cum)=0.205, Q2(cum)=0.189; PC2:R2X(cum) =0.310, Q2(cum)=0.274, PC3:R2X(cum)=0.386, $\mathrm{Q} 2(\mathrm{cum})=0.338$. 3. Supervised PLS-DA modeling with three groups of $\mathrm{Ml}$ cases, non-MI chest pain cases and the controls. $A 3, P C 1: R 2 X(c u m)=0.073$, $\mathrm{R} 2 \mathrm{Y}(\mathrm{cum})=0.339, \mathrm{Q} 2$ (cum) $=0.270 ; \mathrm{PC2}: \mathrm{R} 2 \mathrm{X}$ (cum) $=0.128, \mathrm{R} 2 \mathrm{Y}$ (cum) $=0.544, \mathrm{Q} 2$ (cum) =0.362; PC3:R2X(cum) $=0.174, \mathrm{R} 2 \mathrm{Y}$ (cum) =0.635, Q2(cum) =0.394. Permutation tests with the intercepts of R2<0.23, Q2<-0.20. B3, PC1:R2X(cum) =0.184, R2Y(cum) =0.215, Q2(cum) =0.207; PC2:R2X(cum) =0.249, $\mathrm{R} 2 \mathrm{Y}(\mathrm{cum})=0.400, \mathrm{Q} 2$ (cum) $=0.377, \mathrm{PC3}: \mathrm{R} 2 \mathrm{X}(\mathrm{cum})=0.315, \mathrm{R} 2 \mathrm{Y}(\mathrm{cum})=0.547, \mathrm{Q} 2$ (cum) $=0.512$. Permutation tests with the intercepts of R2<0.10, Q2<-0.05. 4. Supervised PLS-DA modeling with four groups of $M I$ cases $(n=85), U A(n=34)$, other non-MI cardiac cases $(n=27)$ and the controls $(n=84)$. $A 4$, PC1:R2X(cum) =0.073, R2Y(cum) =0.259, Q2(cum) =0.198; PC2:R2X(cum) =0.132, R2Y(cum) =0.397, Q2(cum) =0.269; PC3:R2X(cum)=0.173, $R 2 Y($ cum $)=0.492, Q 2$ (cum) $=0.289$. Permutation test with the intercepts of $R 2<0.180, Q 2<-0.15$. B4,PC1:R2X (cum) $=0.183, R 2 Y($ cum $)=0.169$, 

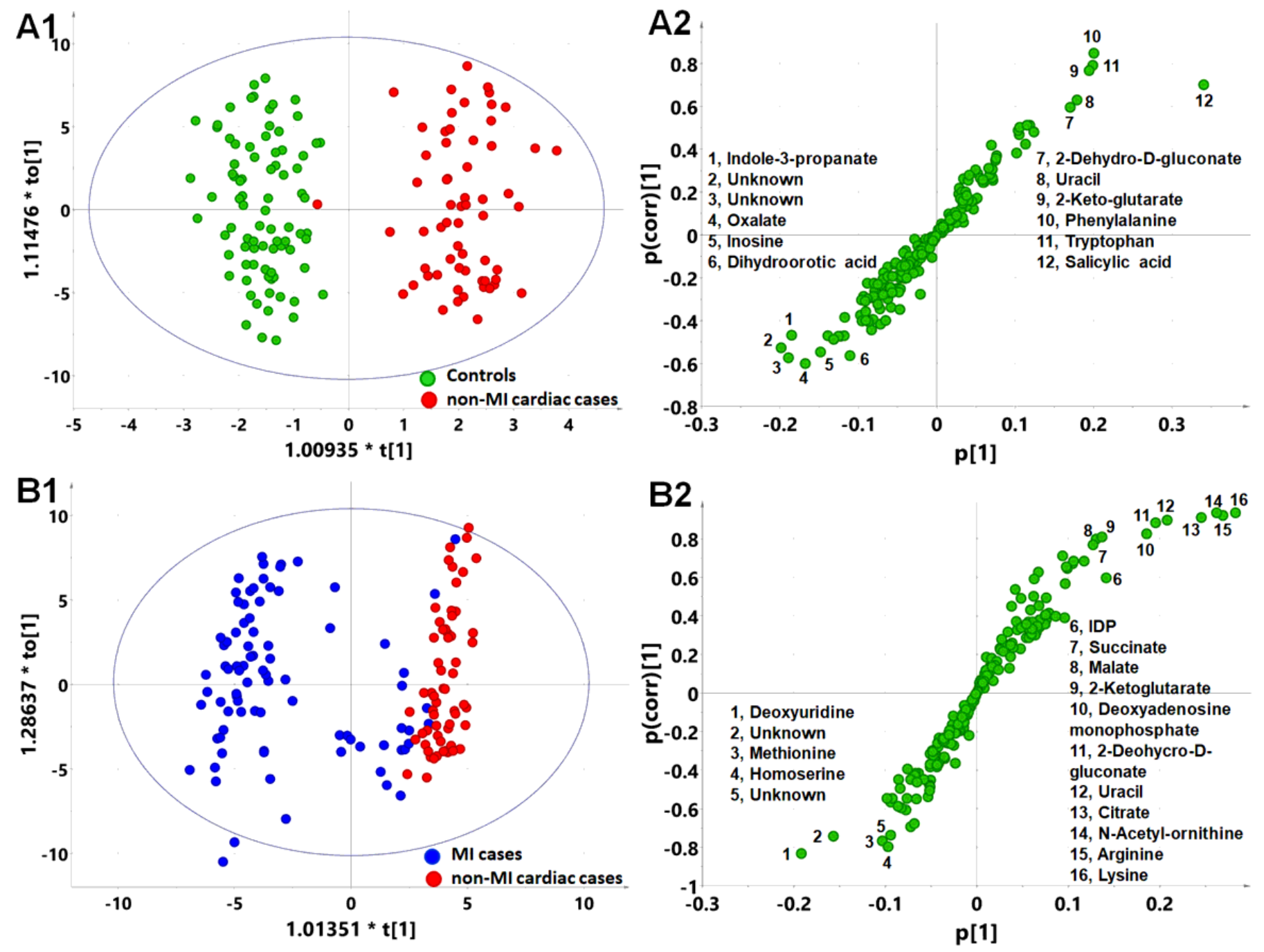

Figure 2

OPLS-DA modeling and S-plots delineate different metabolic phenotypes and potential markers of MI and non-MI cardiac cases. A1, OPLS-DA model differentiating non-MI cardiac cases $(n=61)$ from the controls $(n=84)$; A2, S-plot highlights the potential markers of the non-MI cardiac cases. B1, OPLS-DA model differentiating MI cases $(n=85)$ from non-MI cardiac cases $(n=61)$; B2, S-plot highlights the potential markers of the MI cases. 
A

Heart-related chest pain cases

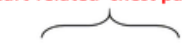

MI cases non-MI chest pain cases

The controls

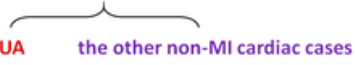

(e.g. myocarditis)

E

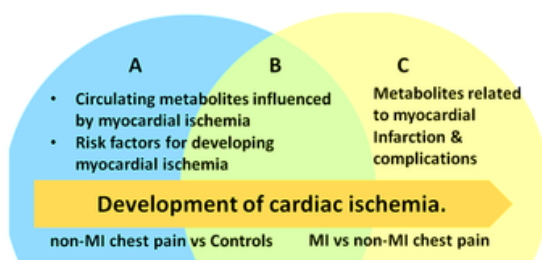

I Matabolites

Methionine

Methionine-adjusted

Homoserine

Homoserine-adjusted

Deoxyuridine

Deoxyuridine-adjusted $\log (\mathrm{OR}) /(95 \% \mathrm{CI})$

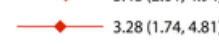

- $\quad 1.61(1.04,2.18$

$\rightarrow \quad 1.61(1.04,2.18)$

$\longrightarrow \quad 3.01(1.71,4.30$

$\longrightarrow \quad 2.66(1.26,4.07)$ $\longrightarrow \quad 3.48(2.01,4.94)$

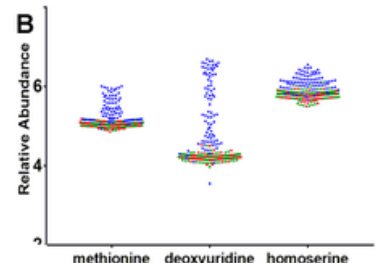

methionine deoxyuridine homoserine
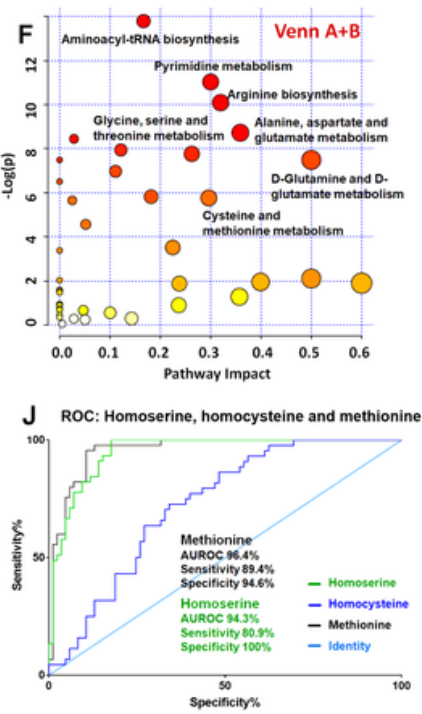
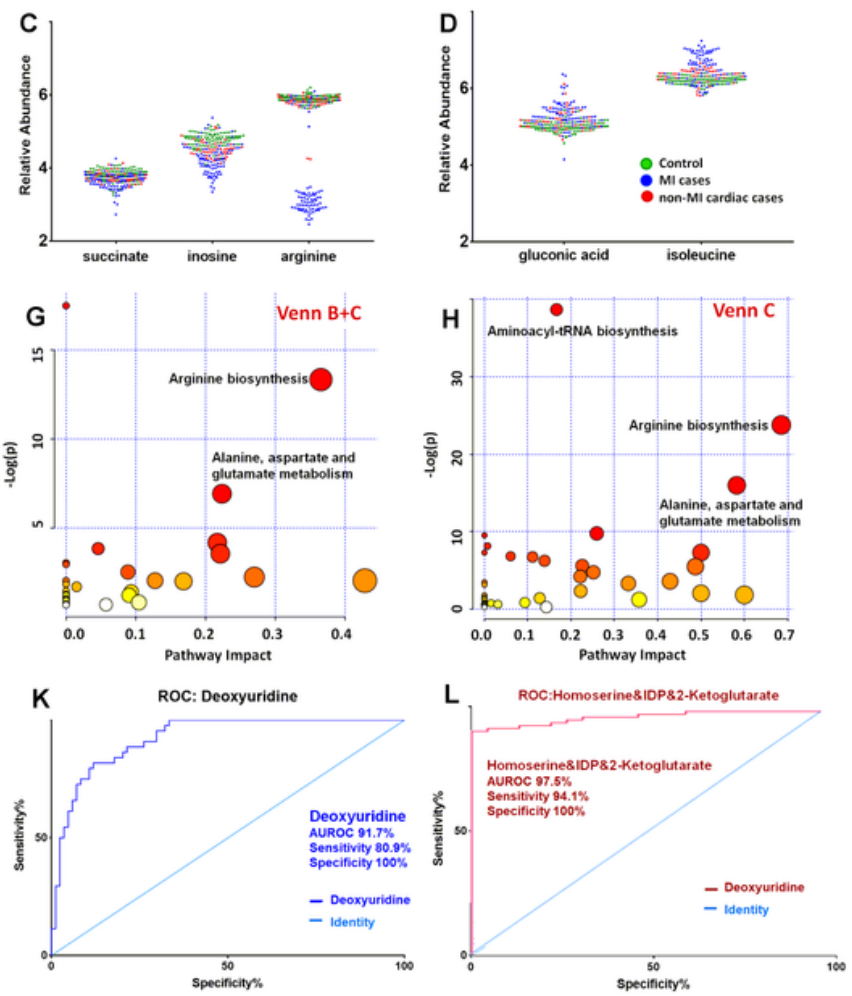

Figure 3

Differential metabolites and pathways involved in MI group A) Samples are divided into 3 groups: control( $(n=84)$, MI(n=85) cases and non-MI chest pain cases $(n=61)$ or 4 groups: control $(n=84), M I$ cases $(n=85), U A(n=34)$ and the other non-MI cardiac cases( $n=27)$ when OPLS-DA analysis is applied. B) Plasma methionine, deoxyurdine and homoserine levels spike in Ml group. C) Succinate, inosine and arginine levels drop as CAD progresses to MI. D) Gluconic acid and isoleucine levels increase as CAD progresses to MI. E) Venn diagram shows discriminant metabolites can be classified into region A,B and C. F) Pathway analysis of differential metabolites in Venn A\&B region (control vs non-MI chest pain cases) G) Pathway analysis of differential metabolites in Venn C\&B (MI vs non-MI cases). There is remarkable change of arginine biosynthesis and alanine,aspartate, glutamate metabolism. . $\mathrm{H}$ ) Pathway analysis of differential metabolites in Venn $\mathrm{C}$. There is remarkable change of arginine biosynthesis and alanine,aspartate, glutamate metabolism. . I) Unadjusted and adjusted (adjusted for age, gender, LDL-c, HDL-c, smoking/diabetic/hypertensive history) odd ratio of methionine, deoxyurdine and homoserine. J) ROC analysis of homoserine (AUC 94.31\%, sensitivity $80.9 \%$, specificity $100 \%$ ), methionine(AUC $96.43 \%$, sensitivity $89.4 \%$, specificity $94.59 \%$ ), homocysteine(AUC $72.78 \%$ ); K) ROC analysis of deoxyuridine (AUC $91.66 \%$, sensitivity $80.9 \%$, specificity $100 \%$ ); L) ROC analysis of a combination of homoserine, IDP and 2-ketoglutarate (AUC=97\%, sensitivity $94.12 \%$, specificity $100 \%$ ). 

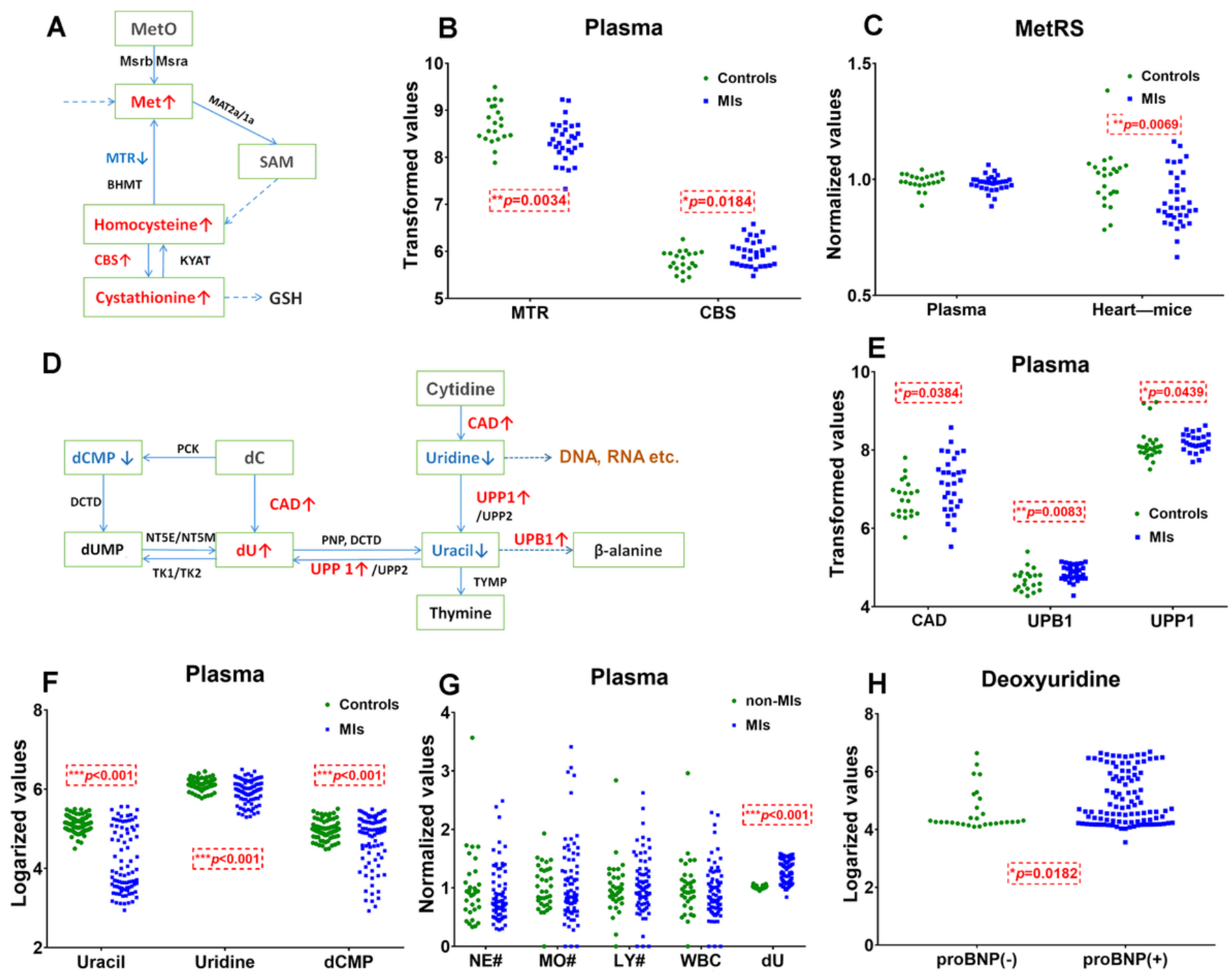

Figure 4

Methionine and dU related pathway in plasma A. Methionine(Met) abundance related pathway in plasma B. Met related enzymes MTR is significantly* decreased and CBS is significantly higher in MI cases(n=31) plasma compared to healthy individuals(n=21). C. Met related enzymes MetRS(MARS2) abundance in myocardium of murine MI models $(n=36)$ is down-regulated* compared to control( $(n=23)$ while human plasma MetRS remain unchanged. D. dU abundance related pathway in plasma E. dU related enzymes CDA, UPB1 and UPP1 are significantly* higher in MI group( $\mathrm{n}=31)$ plasma compared to healthy individuals $(n=21)$. F. dU related intermediates uracil, uridine and dCMP significantly* dropped in MI cases $(n=85)$ compared to healthy individuals(n=84). G. Neutrophils, monocytes, lymphocytes and white cell counts of non-MI cases(n=34) and MI cases(n=74). H. dU is significantly* higher in NT-proBNP(+) group $(n=104)$ than in NT-proBNP(-) group $(n=29)$. (Panel A and D: red indicates up-regulated metabolites or enzymes, blue indicates the down-regulated, black indicates the unchanged and gray indicates undetected metabolites. *Student's t-test $\ \mathrm{p}<0.05$ )

\section{Supplementary Files}

This is a list of supplementary files associated with this preprint. Click to download.

- Supplementaryfigures.docx

- Supplementarymethods.docx

- Supplementarytables.docx 